\title{
The Phase-II ATLAS Pixel Tracker Upgrade: Layout and Mechanics
}

\author{
Abhishek Sharma, CERN \& University of Oxford
}

\begin{abstract}
The ATLAS experiment is planning a major upgrade of its tracking detectors during the Phase-II LHC shutdown, to better take advantage of the increased luminosity of the HL-LHC. The layout of the Pixel Tracker is undergoing an optimisation and will be finalised in 2017. The various layouts are presented. A description of the supporting structures, along with results from testing of prototypes are also discussed.
\end{abstract}

\section{INTRODUCTION}

The ATLAS experiment [1] will be upgrading its tracking detectors during the Phase-II LHC shutdown period in order to maintain the physics performance in view of the increase of luminosity expected in the High Luminosity LHC (HLLHC) era. The upgraded tracker [2] will consist of silicon strip modules surrounding a pixel detector, and is likely to cover an extended eta range, possibly as far as $|\eta|<$ 4.0. Several layout and supporting-structure options will be considered for the pixel detector, the final choice of which is expected to be made in 2017. The proposed supporting structures will be based on lightweight, highly thermally conductive carbon-based materials and cooled by evaporative carbon dioxide.

This paper discusses the various layouts, and a description of the supporting structures are presented, along with results from testing of prototypes.

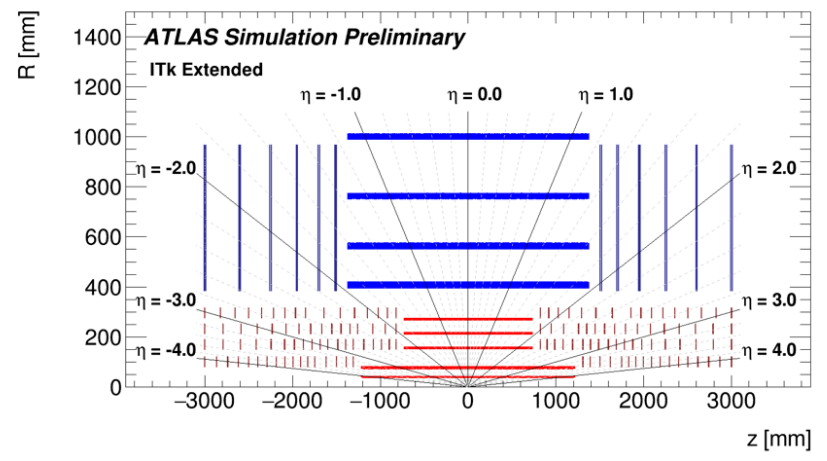

Fig. 1: Layout Concept: Extended 4.0 [2]

\section{LAyout \& Stave Options}

The barrel region will be composed of rectangular modules mounted on long staves, arranged as five concentric cylinders as shown on figure 1 . The modules are expected to be mounted parallel with the beampipe at least in the very central barrel region, with some modules possibly tilted

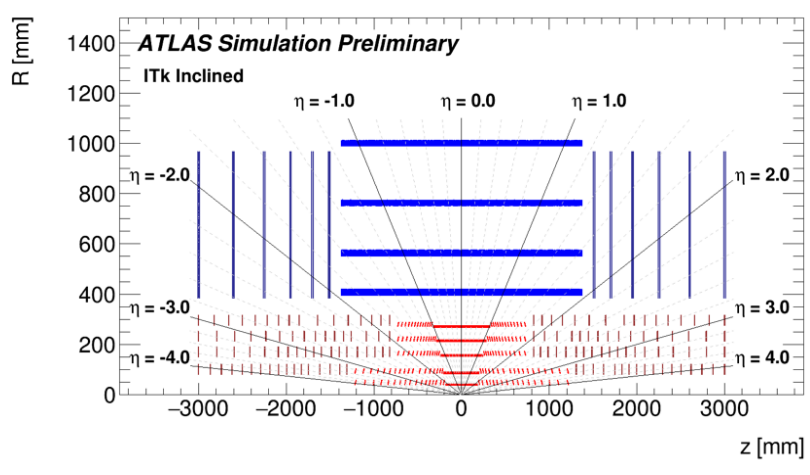

Fig. 2: Layout Concept: Fully Inclined 4.0 [2]

with respect to the beampipe at larger eta, to keep them more normal to tracks coming from the interaction point as shown on figure 2 . The endcap region will be composed of a series of rings arranged in different planes perpendicular to the beam. The coverage of the detector would reach up to $|\eta|<4$. A main component of the Pixel detector are the staves. These are support structures which secure not only the sensors but also their relevant services such as read out cables, cooling pipes and power cables. Four different barrel stave prototypes are being considered: the SLIM Truss, SLIM Longeron, I-Beam and Alpine. See figure 3.

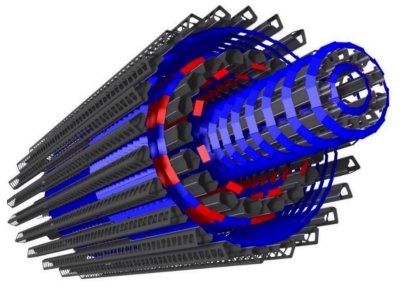

(a) Longeron.

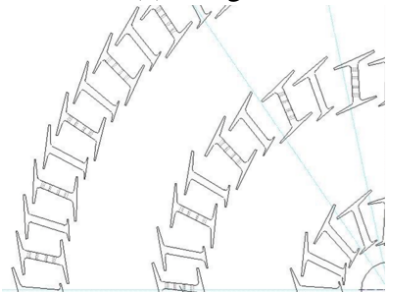

(c) I-Beam.

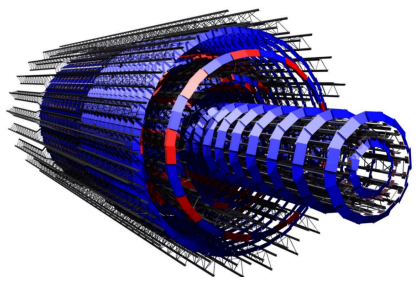

(b) Truss.

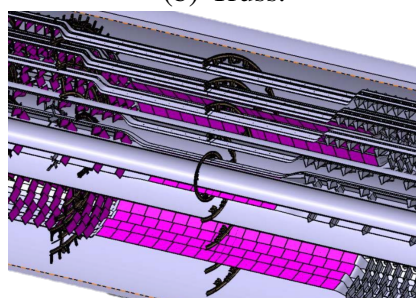

(d) Alpine.
Fig. 3: Four prototype stave options suitable for both tilted and flat layouts. 
The SLIM staves would be supporting individual cells, as shown on figure 4 , on the surface of which modules would be placed.

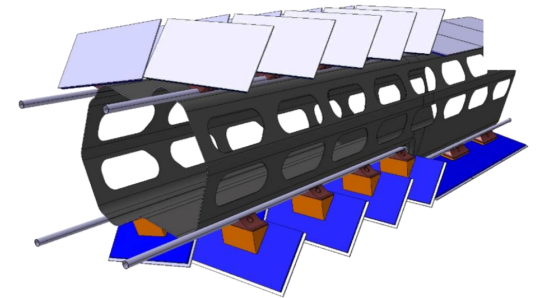

Fig. 4: A Longeron Stave supporting four rows of tilted barrel cells and flat barrel cells in the central part of the detector.

\section{Mechanical Performance Studies}

A variety of mechanical simulation studies have been conducted on each stave type. These include extensive modal analyses as shown for the SLIM Truss on figure 5, in the case of which excellent performance has been observed regardless of angular position. Simulations include single constraint and fully constraint scenarios. A similar modal analysis was conducted for the Endcap ring stave as well, shown below.

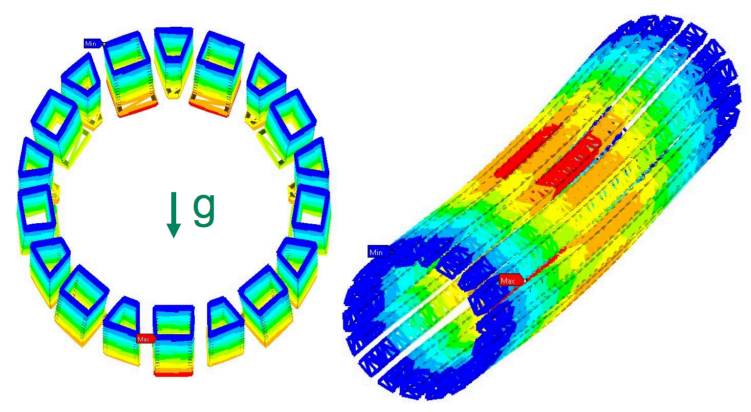

(a) Truss staves.

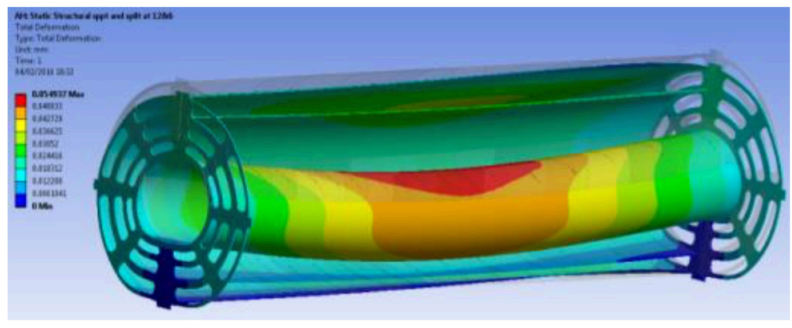

(b) Barrel and End-cap ring regions.

Fig. 5: Modal analyses conducted on various staves.

Optimisation of routing scenarios for sensor services has been an area of significant study. An optimal cable layout is essential for effective operation and ease of maintenance of the staves and these must be taken into account during feasibility studies.

Powering and data I/O connections are achieved either by stave flexes, cabling, or a combination of both as shown on figure 6. Using stave flexes allows for a significant reduction

in the materials present within the tracker volume. On the other hand, tilted cell layout schemes require a doublefolding of flexes which in turn increases the complexity of executing this method.

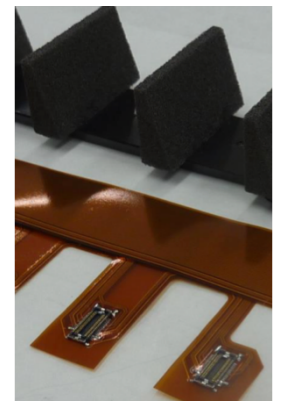

(a) Alpine flex stave.

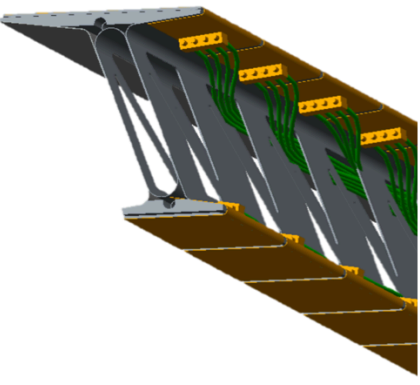

(b) I-Beam routing CAD.

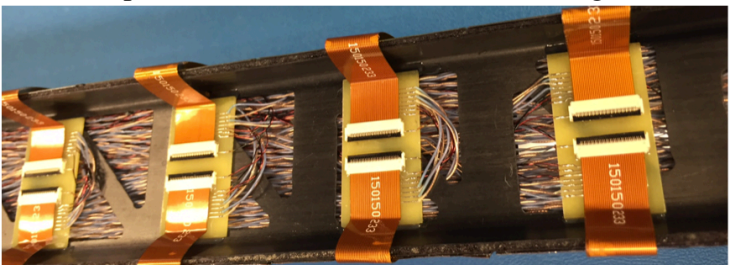

(c) Physical sample of I-Beam with services cabling.

Fig. 6: Data I/O and services cable routing technologies.

Simulations, see figure 7, are complemented by physical testing of stave prototype samples, discussed in the next section.
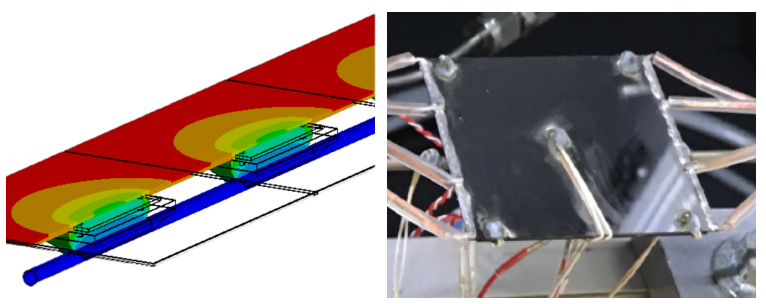

(a) SLIM Barrel cell FEA(b) SLIM Barrel cell physical simulation. measurement.

Fig. 7: Thorough simulation-based analyses complemented by physical measurements have been undertaken.

\section{Physical Measurements of Stave Thermal PERFORMANCE}

The physical measurements undertaken to characterise the thermal performance of these prototypes staves involve measuring their Thermal Figure Of Merit (TFM). This parameter is defined as:

$$
\text { TFM }=\frac{T_{\text {hot }}-T_{\text {cold }}}{\text { Power/Area }}\left[\frac{K \mathrm{~cm}^{2}}{W}\right]
$$

Here $T_{\text {hot }}$ corresponds to the worst cooled point on the surface being studied and $T_{\text {cold }}$, the corresponding $\mathrm{CO}_{2}$ cooling temperature under that location along the length of the pipe. 
$100 \mathrm{~K} \Omega$ NTCs are positioned at the inlet and outlet of the $\mathrm{CO}_{2}$ cooling pipe which allows for a linear interpolation to be made between the cold temperature at various points along the stave's surface. All NTCs are secured onto the stave surfaces using the very high thermal conductivity glue, Artic Silver ASTA7G.
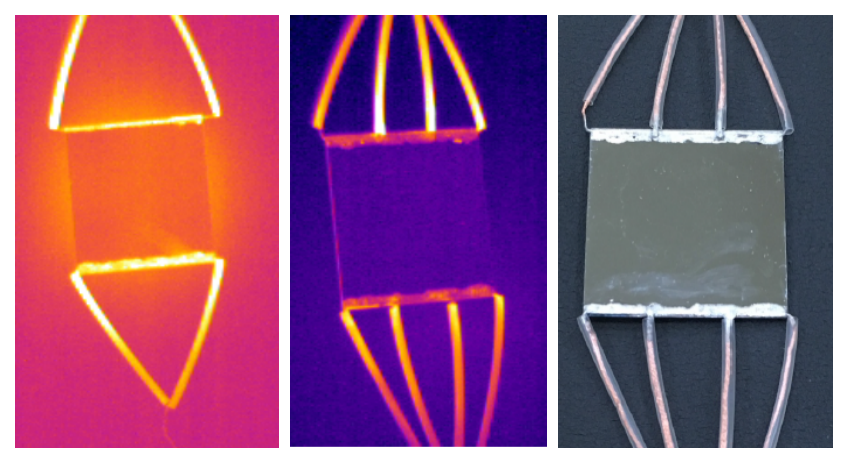

Fig. 8: Copper-wire braiding iterations.

A $350 \mu \mathrm{m}$ thin silicon heater is used to simulate the power dissipation each stave would experience during HLLHC operation. This is estimated to be a heat flux rate of $0.7 \mathrm{~W} / \mathrm{cm}^{2}$. TFM measurement results are hence always quoted at a heat flux value of $0.7 \mathrm{~W} / \mathrm{cm}^{2}$. These silicon heaters have undergone significant improvements in order to deliver heat uniformly across the entirety of the surface area being studied on each particular stave. The initial two-wire copper-braid approach yielded non-negligible inhomogeneity across such surfaces as shown in figure 8 . This effect was considerably reduced by the now optimum combination of four-point copper-braid connections in between every silicon heater cell. In order to secure the copper braids onto the silicon heaters without causing any heat-induced damage to the predominantly carbon-fiber-based prototypes, a very low temperature "Wood-Metal" soldering agent was used.

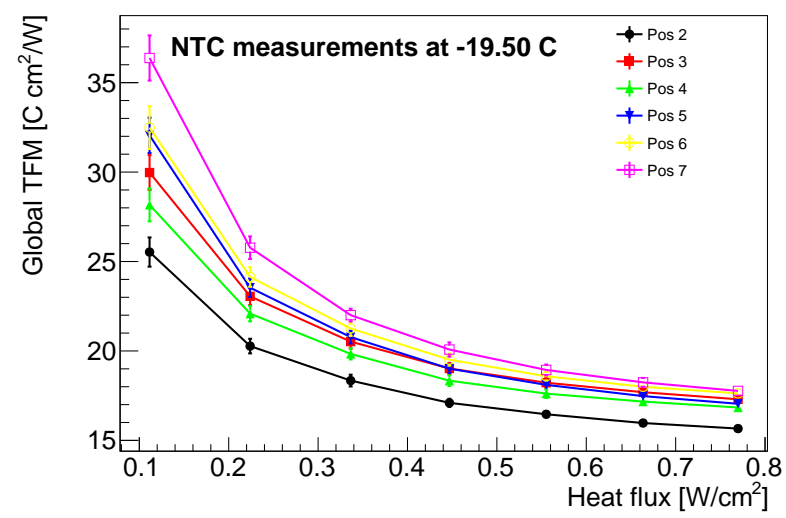

Fig. 9: TFM measurement results for I-Beam (wide side).

Figure 9 shows a measurement result for an I-Beam prototype stave where a TFM value of $17.5 \pm 0.6 \mathrm{Kcm}^{2} / \mathrm{W}$ was obtained, well below the $20 \mathrm{Kcm}^{2} / \mathrm{W}$ required for layer two of the ITK barrel. TFM values are expected to stay constant over varying heat fluxes. The higher values seen above at low heat fluxes are due to convective heat pickup from the environment. As the heat flux onto the stave is increased, this effect becomes increasingly negligible.

\section{Conclusions}

Studies are ongoing to evaluate the performance of different staves under consideration for the ATLAS pixel upgrade. These studies are still at an early stage. A full comparison of the various layouts is also ongoing and a decision on which one will be implemented for the Phase II ITK pixel Upgrade is expected by 2017 .

\section{REFERENCES}

[1] ATLAS Collaboration, 2008 JINST 3 S08003.

[2] ATLAS Collaboration, Expected performance of the ATLAS Inner Tracker at the High-Luminosity LHC, ATL-PHYS-PUB-2016-025. 